\title{
Both the Duration of Apnea and the Number of Apneas Are Important in Obstructive Sleep Apnea Syndrome
}

\author{
Jae Hoon Cho, MD, MhD \\ Department of Otorhinolaryngology-Head and Neck Surgery, College of Medicine, Konkuk University, Seoul, Korea
}

\begin{abstract}
Apnea and hypopnea are generally considered to be equivalent in calculating the apnea-hypopnea index (AHI) regardless of their kind and duration. However, we supposed that apnea and hypopnea could differ in their effects on arousal and oxygen desaturation, and that the duration of an occurrence of apnea or hypopnea could also be important. The following were analyzed for 182 cases of polysomnography in obstructive sleep apnea (OSA) patients. 1) We calculated the correlations between variables, such as AHI, arousal index, oxygen desaturation index (ODI), apnea index (AI), hypopnea index (HI), and mean apnea duration, using common statistical procedures. 2) We compared AHI, arousal index, and ODI in patients divided into short and long mean apnea-duration groups. We found that 1) AHI, arousal index, ODI, mean apnea duration, and AI were highly correlated with each other, but HI was not; and 2) arousal index and ODI were higher in the long mean apnea-duration group than in the short mean apnea-duration group. Hence apnea is more important for arousal and oxygen desaturation during sleep than is hypopnea. Longer apnea duration is associated with both more severe OSA and greater arousal and oxygen desaturation.
\end{abstract}

Sleep Med Res 2020;11(2):149-151

Key Words Obstructive sleep apnea syndrome, Apnea-hypopnea index, Apnea, Hypopnea.

\section{INTRODUCTION}

Apnea and hypopnea frequently cause oxygen desaturation and arousal in obstructive sleep

Received: November 5, 2020

Revised: December 7, 2020

Accepted: December 12, 2020

Correspondence

Jae Hoon Cho, MD, MhD

Department of Otorhinolaryngology-

Head and Neck Surgery,

Konkuk University Hospital,

120-1 Neungdong-ro, Gwangjin-gu,

Seoul 05030, Korea

Tel +82-2-2030-7667

Fax +82-2-2030-5299

E-mail jaehoon@kuh.ac.kr

\section{ORCID}

Jae Hoon Cho

https://orcid.org/0000-0002-2243-7428

(c) This is an Open Access article distributed un der the terms of the Creative Commons Attribution Non-Commercial License (https://creativecommons.org/licenses/by-nc/4.0) which permits unrestricted non-commercial use, distribution, and reproduction in any medium, provided the original work is properly cited. apnea (OSA) syndrome. These oxygen desaturations and arousals have been well known to trigger various cardiovascular complications. The oxygen desaturation provokes pulmonary vasoconstriction and impairment of cardiac contractability. Moreover, intermittent oxygen desaturation induces oxygen-free-radical production, which can activate inflammation and impair endothelial function. Arousal increases sympathetic tone and suppresses the vagal tone, which sustains high blood pressure and rapid heart rate. All those effects play an important role in the occurrence and aggravation of various cardiovascular diseases, such as hypertension, arrhythmia, stroke, and heart attack [1-5].

The number of apneas and hypopneas is well correlated with the number of oxygen desaturations and arousals; therefore they can be important parameters for evaluating the patient's status and predicting the patient's death. Hypopnea is always followed by oxygen desaturation by definition, whereas apnea is not thus followed. We define apnea as when cessation of breathing lasts longer than 10 seconds [6], but it occurred to us that the longer the duration of apnea is, the more severe its effect on oxygen desaturation and arousal can be. The correlations between apnea-hypopnea index (AHI), oxygen desaturation, and arousal index have already been reported [7], but the duration of apnea has not been considered.

Our purpose was to investigate the influence of the duration of apnea on arousal and oxy- 
gen desaturation.

\section{METHODS}

We enrolled 182 OSA patients [age (mean \pm SD), $45.5 \pm 13.3$ years; all older than 16 years; 150 men, 32 women] in this study. Their body mass index was $26.0 \pm 3.4$. All patients underwent full polysomnography in Konkuk University Hospital, and the minimal value of their AHI exceeded five. This study was exempted by Konkuk University Hospital from Internal Review Board approval because this review did not come within the purview of the board (KUH1110066). We calculated the correlations between variables such as AHI, arousal index, oxygen desaturation index (ODI), apnea index (AI), hypopnea index (HI), and mean apnea duration, and we compared AHI, arousal index, and ODI in patients divided into short and long mean apnea-duration groups.

\section{RESULTS}

We looked at the correlations between $\mathrm{AI}, \mathrm{HI}$, arousal index, and ODI. AI used to be much greater than $\mathrm{HI}$, which means that $\mathrm{AHI}$ is determined mainly by apnea. Even more, AI was more correlated with the arousal index and ODI than was AHI (a mixture of apnea and hypopnea), whereas HI did not display any correlation with arousal index or ODI (Table 1).

When we looked at correlations between mean apnea duration, arousal index, ODI, and AHI, we found that mean apnea duration was highly correlated with the arousal index and ODI (Table 2), which means that the longer the duration of apnea is,

Table 1. Correlations between $\mathrm{AHI}, \mathrm{Al}, \mathrm{HI}$, arousal index, and $\mathrm{ODI}$

\begin{tabular}{lccc}
\hline & AHI & AI & HI \\
& $(31.8 \pm 22.9)$ & $(22.7 \pm 23.1)$ & $(9.3 \pm 7.1)$ \\
\hline Arousal index & $\mathrm{C}=0.647$ & $\mathrm{C}=0.690$ & $\mathrm{C}=-0.049$ \\
$(22.5 \pm 18.4)$ & $\mathrm{p}<0.001$ & $\mathrm{p}<0.001$ & $\mathrm{p}=0.509$ \\
ODI & $\mathrm{C}=0.727$ & $\mathrm{C}=0.730$ & $\mathrm{C}=0.138$ \\
$(37.0 \pm 30.3)$ & $\mathrm{p}<0.001$ & $\mathrm{p}<0.001$ & $\mathrm{p}=0.062$ \\
\hline
\end{tabular}

AHI: apnea-hypopnea index, AI: apnea index, HI: hypopnea index, ODI: oxygen desaturation index, C: correlation coefficient, $($ mean $\pm S D)$.

Table 2. Correlations between mean apnea duration, arousal index, ODI, and $\mathrm{AHI}$

\begin{tabular}{lccc}
\hline & $\begin{array}{c}\text { Arousal index } \\
(22.5 \pm 18.4)\end{array}$ & $\begin{array}{c}\text { ODI } \\
(37.0 \pm 30.3)\end{array}$ & $\begin{array}{c}\text { AHI } \\
(31.8 \pm 22.9)\end{array}$ \\
\hline Mean apnea & $\mathrm{C}=0.328$ & $\mathrm{C}=0.360$ & $\mathrm{C}=0.363$ \\
duration & $\mathrm{p}<0.001$ & $\mathrm{p}<0.001$ & $\mathrm{p}<0.001$ \\
$(21.6 \pm 6.9 \mathrm{~s})$ & & & \\
\hline
\end{tabular}

AHI: apnea-hypopnea index, ODI: oxygen desaturation index, C: correlation coefficient, (mean $\pm \mathrm{SD}$ ). the more common the arousal and oxygen desaturation are. Mean apnea duration also was correlated with AHI, which means that as AHI increases, the duration of apnea also increases. When we compared AHI, arousal index, and ODI between the short and long mean apnea-duration groups of patients with mild to moderate OSA, there was no significant difference of AHI or arousal index between the short $(<20 \mathrm{~s})$ and long $(\geq 20 \mathrm{~s})$ mean apnea-duration groups, but ODI was greater in the long mean apnea-duration group (Fig. 1). When we compared AHI, arousal index, and ODI between the short and long mean apnea-duration groups of patients with severe OSA, there was no significant difference of AHI or ODI between the short $(<20 \mathrm{~s})$ and long ( $\geq 20 \mathrm{~s}$ ) mean apnea-duration groups, but the arousal index was greater in the long mean apnea-duration group (Fig. 2).

\section{DISCUSSION}

OSA is characterized by apneic episodes and oxygen desaturation, which are ended by brief arousals and the reestablishment of upper airway patency [1]. Since arousal during sleep and oxygen desaturation usually follow apnea and hypopnea, the arousal index and ODI are closely correlated with AHI $[1,7]$, which is defined as the total number of episodes of apnea and hypopnea per hour of sleep. The criterion for an episode is that it lasts for more than 10 seconds [6]. However, viewing apnea and hypopnea as equivalent, and counting all episodes longer than 10 seconds for both regardless of their duration, is very arbitrary. Since apnea is defined as a decrease in airflow of more than $50 \%$, whereas hypopnea is defined as a decrease of $30-50 \%$ [6], it was natural to suppose that the effects of apnea would be more serious than those of hypopnea. Similarly, it seemed unreasonable that a 10-sec episode of apnea should contribute as much to AHI as does a 50 -second episode.

Not only apnea but also hypopnea are well known to be an independent factor for predicting a grave consequence for OSA patients. However, as shown in this study, usually apnea was much more frequent than was hypopnea; so AHI was mainly composed of the apnea number, and only AI was well correlated with the arousal index and ODI, but HI was not. Moreover, AHI did not increase the correlation more than did AI alone, which means that hypopnea has little effect on arousal and oxygen desaturation. Considering all these findings, it is reasonable to weight apnea more than hypopnea in calculating AHI.

Up to now, it has been thought that severe OSA has a worse prognosis only because the patients with severe OSA experience more episodes of apnea and hypopnea than do patients with mild to moderate OSA. However, our results showed that the duration of apnea increased proportionately in increasing $\mathrm{AHI}$; that is, severe OSA patients experience longer episodes of apnea than do patients with mild to moderate OSA. This fact points to a possible additional reason for worse prognosis of se- 

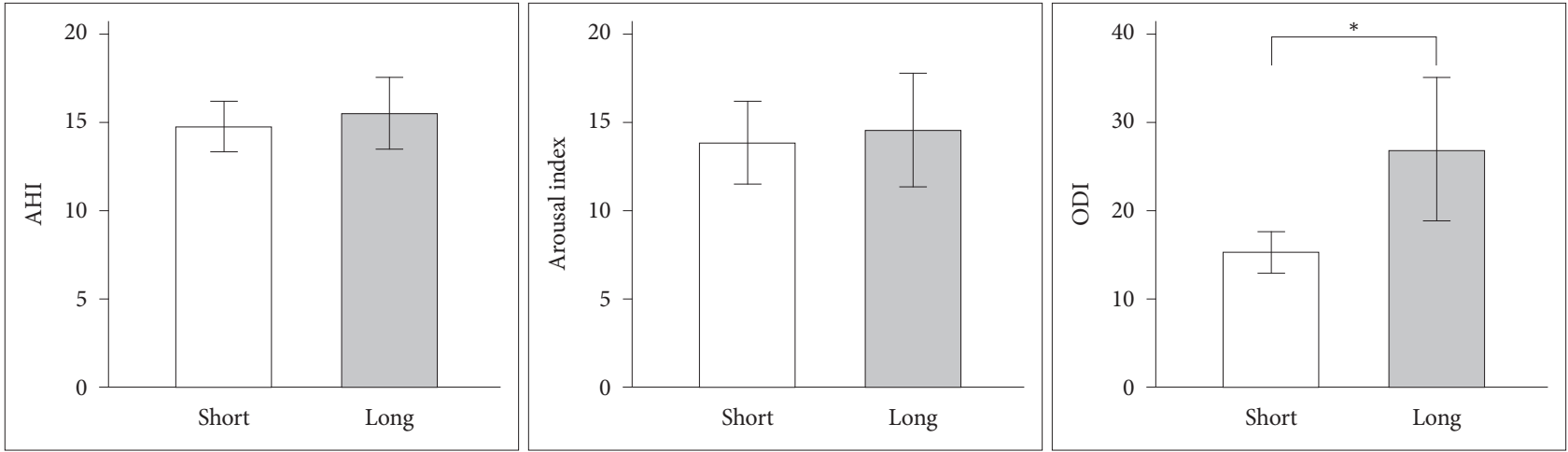

Fig. 1. Comparison of AHIs and arousal indices in mild to moderate sleep apnea patients. ODI was greater in the long mean apnea-duration group $(n=38)$ than in the short mean apnea-duration group $(n=65)(p=0.001) .{ }^{*} p<0.05$. AHI: apnea-hypopnea index, ODI: oxygen desaturation index.
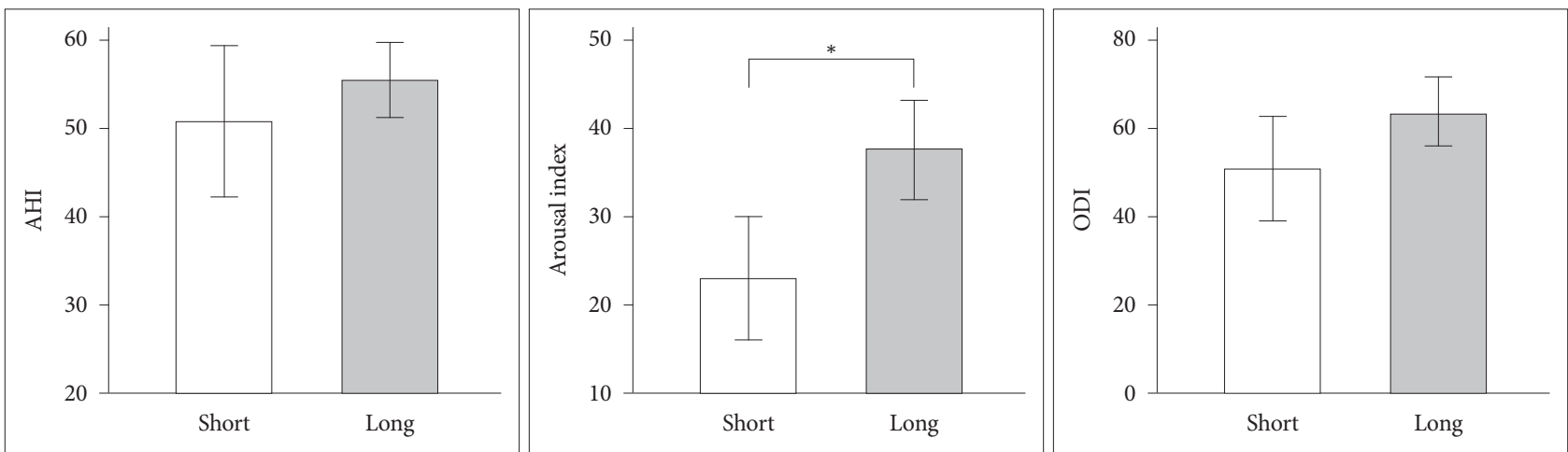

Fig. 2. Comparison of AHIs and arousal indices in severe sleep-apnea patients. The arousal index was greater in the long mean apnea-duration group $(n=57)$ than in the short mean apnea-duration group $(n=22)(p=0.006) .{ }^{*} p<0.05$. AHI: apnea-hypopnea index, ODI: oxygen desaturation index.

vere OSA. We also showed that the longer mean apnea-duration is, the more common the arousal and oxygen desaturation are, which also explains the grave effect of the longer apnea duration.

When we divided episodes of apnea into short versus long duration, with a cut-off value of 20 seconds, we found that, for mild to moderate OSA, ODI (not arousal index) was much greater in the long mean apnea-duration group than in the short duration group, and that, for severe OSA, the arousal index (not the ODI) was much greater in the long mean apnea-duration group than in the short duration group. We do not understand the reason for this difference between mild to moderate and severe OSA, but in any circumstance, the results show that apnea duration is important for arousal and oxygen desaturation.

AHI has proved to be a powerful predictor [6]. However, the effects of apnea and hypopnea may be totally different, and, in addition, the duration of the apnea is relevant. Hence for more accurate prognosis, we may need a novel variable that takes all these factors into account.

In conclusion, apnea is more important for arousal and oxygen desaturation during sleep than hypopnea is. The more severe OSA is, the longer the apnea duration is, and apnea duration is also an important variable for arousal and oxygen desaturation.

\section{Acknowledgments}

None.

\section{Conflicts of Interest}

The author has no financial conflicts of interest.

\section{REFERENCES}

1. Ralls F, Cutchen L. A contemporary review of obstructive sleep apnea. Curr Opin Pulm Med 2019;25:578-93.

2. Seda G, Han TS. Effect of obstructive sleep apnea on neurocognitive performance. Sleep Med Clin 2020;15:77-85.

3. Xia W, Huang Y, Peng B, Zhang X, Wu Q, Sang Y, et al. Relationship between obstructive sleep apnoea syndrome and essential hypertension: a dose-response meta-analysis. Sleep Med 2018;47:11-8.

4. Liu X, Ma Y, Ouyang R, Zeng Z, Zhan Z, Lu H, et al. The relationship between inflammation and neurocognitive dysfunction in obstructive sleep apnea syndrome. J Neuroinflammation 2020;17:229.

5. Prasad B, Nyenhuis SM, Imayama I, Siddiqi A, Teodorescu M. Asthma and obstructive sleep apnea overlap: what has the evidence taught us? Am J Respir Crit Care Med 2020;201:1345-57.

6. Kushida CA, Littner MR, Morgenthaler T, Alessi CA, Bailey D, Coleman J Jr, et al. Practice parameters for the indications for polysomnography and related procedures: an update for 2005. Sleep 2005;28:499521.

7. Pitson DJ, Stradling JR. Autonomic markers of arousal during sleep in patients undergoing investigation for obstructive sleep apnoea, their relationship to EEG arousals, respiratory events and subjective sleepiness. J Sleep Res 1998;7:53-9. 